\title{
Microvascular fluid exchange during pulsatile cardiopulmonary bypass perfusion with the combined use of a nonpulsatile pump and intra-aortic balloon pump
}

\author{
Steinar Lundemoen, MD, ${ }^{\mathrm{a}}$ Venny Lise Kvalheim, MD, PhD, ${ }^{\mathrm{a}, \mathrm{b}}$ Arve Mongstad, RP, ${ }^{\mathrm{a}}$ \\ Knut Sverre Andersen, MD, PhD, ${ }^{\text {a }}$ Ketil Grong, $\mathrm{MD}, \mathrm{PhD},{ }^{\mathrm{b}}$ and Paul Husby, MD, $\mathrm{PhD}^{\mathrm{b}, \mathrm{c}}$
}

Objective: To evaluate how pulsed versus nonpulsed cardiopulmonary bypass influences microvascular fluid exchange in an experimental setup combining a nonpulsatile perfusion pump and an intra-aortic balloon pump.

\begin{abstract}
Methods: A total of 16 pigs were randomized to pulsatile cardiopulmonary bypass perfusion with an intra-aortic balloon pump switched to an automatic 80 beats/min mode after the start of cardiopulmonary bypass (pulsatile perfusion $[\mathrm{PP}]$ group, $\mathrm{n}=8$ ) or to nonpulsatile cardiopulmonary bypass with the pump switched to the off position (nonpulsatile $[\mathrm{NP}]$ group, $\mathrm{n}=8$ ). Normothermic cardiopulmonary bypass was initiated after 60 minutes of stabilization and continued for 3 hours. The fluid needs, plasma volume, colloid osmotic pressure in plasma, colloid osmotic pressure in interstitial fluid, hematocrit, and total tissue water content were recorded, and the protein masses and fluid extravasation rates were calculated.
\end{abstract}

Results: After cardiopulmonary bypass was started, the mean arterial pressure increased in the PP group and decreased in the NP group. At 180 minutes, the mean arterial pressure of the PP and NP groups was $70.9 \pm$ $2.7 \mathrm{~mm} \mathrm{Hg}$ and $55.9 \pm 2.7 \mathrm{~mm} \mathrm{Hg}$, respectively $(P=.004)$. The central venous pressure (right atrium) had decreased in the NP group $(P=.002)$. A decreasing trend was seen in the PP group. No between-group differences were present. The hematocrit and colloid osmotic pressure in plasma and interstitial fluid had decreased similarly in both study groups during cardiopulmonary bypass. The plasma volume of the PP group had decreased initially but then returned gradually to precardiopulmonary bypass levels. In the NP group, the plasma volume remained contracted $(P=.02)$. No significant differences in the fluid extravasation rate were obtained. The fluid extravasation rate of the PP group tended to stay slightly higher than the fluid extravasation rate of the NP group at all measurement intervals. The total tissue water content increased significantly in a number of organs compared with that in the control animals. However, differences in the total tissue water content between pulsed and nonpulsed perfusion were absent.

Conclusions: No significant differences in the fluid extravasation rates were present between pulsed and nonpulsed cardiopulmonary bypass perfusion in the present experimental setup. (J Thorac Cardiovasc Surg 2013;146:1275-82)

The use of cardiopulmonary bypass (CPB) during heart surgery has been associated with fluid accumulation that can interfere with vital organ function. ${ }^{1,2}$ Increased microvascular fluid filtration and/or impaired lymphatic drainage have been the reported culprits.

The factors responsible for an increase in microvascular fluid filtration include crystalloid hemodilution with a decrease in plasma oncotic pressure, ${ }^{3}$ an increase in the

\footnotetext{
From the Section for Cardiothoracic Surgery, ${ }^{\mathrm{a}}$ Department of Heart Disease and Department of Anesthesia and Intensive Care, ${ }^{\mathrm{c}}$ Haukeland University Hospital, Bergen, Norway; and Department of Surgical Sciences, ${ }^{\mathrm{b}}$ University of Bergen, Bergen, Norway. The present study was supported by the Western Norway Regional Health Authority (Stavanger, Norway) and the Grieg Foundation (Bergen, Norway).

Disclosures: Authors have nothing to disclose with regard to commercial support. Received for publication Oct 10, 2012; revisions received April 29, 2013; accepted for publication June 14, 2013; available ahead of print Aug 1, 2013.

Address for reprints: Paul Husby, MD, PhD, Department of Anesthesia and Intensive Care, Haukeland University Hospital, Bergen N-5021, Norway (E-mail: paul. husby@kir.uib.no).

$0022-5223 / \$ 36.00$

Copyright (c) 2013 by The American Association for Thoracic Surgery

http://dx.doi.org/10.1016/j.jtcvs.2013.06.002
}

central venous pressure (CVP), with elevation of the capillary hydrostatic pressure $(\mathrm{Pc}),{ }^{4}$ the presence of a generalized inflammatory reaction related to contact between the blood and foreign surfaces of the CPB circuit, ${ }^{5,6}$ hypothermic and tepid conditions during $\mathrm{CPB},{ }^{1,2}$ the $\mathrm{CPB}$ perfusion flow rate $^{7}$ the use of volatile anesthetics, ${ }^{8}$ and, finally, the $\mathrm{CPB}$ flow pattern (ie, pulsatile vs nonpulsatile perfusion). ${ }^{9}$

In a previous study of the fluid exchange in the lungs, fluid filtration, expressed as the filtration coefficient, $\mathrm{K}_{\mathrm{f}}$, was found to be higher during pulsatile than nonpulsatile perfusion. ${ }^{9}$ Other investigators have argued that pulsatile perfusion inhibits edema formation. ${ }^{10}$ This contention seems, however, to have been less studied in experimental models.

The optimal perfusion mode during CPB and cardiac surgery remains debated. Although pulsatile perfusion has been regarded as the most physiologic, nonpulsatile CPB perfusion is still a simpler compromise, with few disadvantages.

Recently, a number of studies have focused on the beneficial effects of preoperative intra-aortic balloon pump (IABP) deployment in high-risk patients undergoing 


$$
\begin{aligned}
& \text { Abbreviations and Acronyms } \\
& \text { COP }=\text { colloid osmotic pressure } \\
& \mathrm{COP}_{\mathrm{i}}=\text { COP in interstitial fluid } \\
& \mathrm{CPB}=\text { cardiopulmonary bypass } \\
& \mathrm{CVP}=\text { central venous pressure } \\
& \mathrm{EEP}=\text { energy equivalent pressure } \\
& \mathrm{FER}=\text { fluid extravasation rate } \\
& \mathrm{IABP}=\text { intra-aortic balloon pump } \\
& \mathrm{MAP}=\text { mean arterial pressure } \\
& \mathrm{NFB}=\text { net fluid balance } \\
& \mathrm{NP}=\text { nonpulsatile perfusion } \\
& \mathrm{Pc}=\text { capillary hydrostatic pressure } \\
& \mathrm{PP}=\text { pulsatile perfusion } \\
& \mathrm{PV}=\text { plasma volume } \\
& \mathrm{SHE}=\text { surplus hemodynamic energy } \\
& \mathrm{SVR}=\text { systemic vascular resistance } \\
& \mathrm{TTW}=\text { total tissue water content }
\end{aligned}
$$

coronary artery bypass grafting. ${ }^{11-13}$ Also, the continuation of IABP-pulsed perfusion during CPB has been advocated, because it improves whole body perfusion, preserves endothelial nitric oxide release, and reduces the coagulative and fibrinolytic response. ${ }^{14-16}$ In addition, such a combined perfusion technique contributes to better preservation of the lungs,${ }^{17}$ the splanchnic organs, and the kidneys. ${ }^{13}$

To the best of our knowledge, no randomized clinical or experimental trials have evaluated microvascular fluid homeostasis when pulsed CPB flow is generated with an IABP in automatic 80 beats/min mode.

In the present experimental study, we, therefore, explored in more detail how pulsed versus nonpulsed CPB perfusion influences microvascular fluid exchange when combining a nonpulsatile perfusion pump and IABP.

\section{METHODS \\ Animal Handling, Anesthesia, and Surgical Preparation}

Domestic pigs of either gender, about 3 months old, were studied (Norwegian Landrace Norhybrid; Agriculture College, Stend, Norway). All the pigs were acclimatized within the laboratory housing facility before the experiments. The local laboratory animal veterinarian under surveillance of the Norwegian Animal Research Authority approved the anesthetic and experimental protocol, which was conducted in accordance with the national and international laws and regulations.

All the pigs were fasted overnight but had continuous free access to water.

Anesthesia included midazolam, fentanyl, and pancuronium infusion and isoflurane inhalation. ${ }^{18}$ The heart rate was obtained using surface electrocardiographic electrodes. The CVP and pulmonary arterial and systemic arterial pressures were monitored continuously by catheters introduced into the right atrium, right femoral vein, pulmonary artery, and right mammary artery.

In all pigs, a 7.5F, 25-mL IABP (Datascope Corp, Fairfield, NJ) was inserted by way of the left femoral artery to the radiographically confirmed correct position in the descending aorta with the catheter tip at the level of the aortic valve, with the aim of generating pulsatile flow during CPB.
With reference to Undar, ${ }^{19}$ the energy equivalent pressure (EEP) and surplus hemodynamic energy (SHE) were calculated. The calculations were determined from measured flow in the initial segment of the right carotid artery (Transit-time flowmetry; Med-Stim AS, Oslo, Norway) and the pressure obtained close to the aortic arch (Millar catheter, MPC-500; Millar Instruments Inc, Houston, Tex) to ensure the IABP resulted in adequate and maintained pulsatile $\mathrm{CPB}$ perfusion throughout the 3 hours of $\mathrm{CPB}$. The actual measurements were performed continuously and tracing was done every 30 minutes.

Preparation for extracorporeal circulation was done after intravenous administration of heparin (6 mg/kg plus $3 \mathrm{mg} / \mathrm{kg}$ after 1 hour). An 18F aortic arch cannula (18F EOPA; Medtronic Inc, Minneapolis, Minn) was inserted into the ascending aorta, and a 29F 3 -stage venous return cannula (Medtronic $29 \mathrm{~F}, \mathrm{MC} 2 \mathrm{X} 91429$ ) was placed in the inferior vena cava by way of the right atrium and connected to standard equipment for open heart surgery (Quadrox-I Adult microporous membrane oxygenator, VHK 2000 venous hard-shell reservoir, and standard HLM tubing equipped ow Centrifflow Centrifugal Pump, RF32; Maquet Cardiopulmonary AG, Hirrlingen, Germany).

After the surgical preparation, the pigs were allowed 60 minutes of stabilization before the start of $\mathrm{CPB}$.

The CPB circuit was primed with $1220 \mathrm{~mL}$ of acetated Ringer's solution, resulting in filling of the venous reservoir to the $400 \mathrm{~mL}$ level. The pump flow was $100 \mathrm{~mL} / \mathrm{kg}$, the CPB pump head pressure was 200 to 250 $\mathrm{mm} \mathrm{Hg}$, and the flow pattern was nonpulsatile.

A total of 16 pigs were divided by block randomization into pulsatile perfusion (PP group; $\mathrm{n}=8$ ) or nonpulsatile perfusion (NP group; $\mathrm{n}=8$ ). In the PP group, the IABP was switched to an automatic 80 beats/min mode immediately after the initiation of CPB. For the NP group, the pump remained in the turned off position. Once receiving the calculated flow, ventricular fibrillation and, thus, circulatory arrest were induced with a 9V DC current applied to the right ventricle.

\section{Blood Sampling and Analysis}

Analysis of the hematocrit, serum total protein, serum total albumin, serum total electrolyte concentrations, serum osmolality, and acid base parameters were performed on blood sampled from an arterial line. ${ }^{1}$

\section{Blood and Plasma Volume Determination (Carbon Monoxide Method)}

The red blood cell volume was determined just before the initiation of $\mathrm{CPB}$ using the carbon monoxide method. ${ }^{20}$ Subsequently, the blood volume and plasma volume (PV) were calculated from the determination of the changes in the hematocrit and corrected for blood loss. To assess the real $\mathrm{PV}$ within the pig during $\mathrm{CPB}$, the volumes were corrected for the actual fluid volume present in the extracorporeal circuit at different intervals according to the following formula: in vivo PV = calculated PV $-\mathrm{PV}$ of the $\mathrm{CPB}$ circuit.

\section{Albumin Mass, Protein Mass, and Albumin and Protein Extravasation}

The total (ie, within the pig, tubing, and machine reservoir) intravascular albumin and protein masses (in grams) were calculated as the product of the measured plasma volume (within the pig, tubing, and reservoir; in liters) and the serum albumin or serum protein concentrations in grams per liter. Extravasation or intravasation of albumin and protein were calculated as the change in the albumin and protein mass from one moment to another.

\section{Fluid Loss and Supplementation, Net Fluid Balance, and Fluid Extravasation Rate}

The urine output was recorded every 30 minutes through a suprapubic catheter. The fluid balance (ie, fluid input and output) was recorded continuously.

Maintenance fluid consisted of a continuous infusion of acetated Ringer's solution $(5 \mathrm{~mL} / \mathrm{kg} / \mathrm{min})$. Blood loss before heparinization and 
CPB cannulation was measured by weighing the surgical sponges and replaced by acetated Ringer's solution in volumes 3 times the measured blood loss volume.

Blood loss into the open chest after cannulation and during CPB was returned by suction to circulation through the extracorporeal machine reservoir.

During CPB, the 400-mL level of the machine reservoir was closely monitored and recorded at 5-minute intervals. Changes from the 400-mL level indicated loss or gain of fluid between the circulation and interstitial space or changes in the vascular tone. Whenever the level of the reservoir decreased, acetated Ringer's solution was supplemented to restore the 400-mL level.

The net fluid balance (NFB; ie, all fluid additions and losses, including diuresis and bleeding) was used, together with the changes in PV from one interval to another $(\Delta \mathrm{PV})$ to calculate the FER at the different periods reported, according to the formula:

$$
\operatorname{FER}(\mathrm{mL} / \mathrm{kg} / \mathrm{min})=\mathrm{NFB}(\mathrm{mL} / \mathrm{kg} / \mathrm{min})-\Delta \mathrm{PV}(\mathrm{mL} / \mathrm{kg} / \mathrm{min})
$$

\section{Colloid Osmotic Pressure}

The colloid osmotic pressure (COP) was measured in the plasma and interstitial fluid $\left(\mathrm{COP}_{\mathrm{i}}\right) .{ }^{1}$ Subcutaneous interstitial fluid sampling was performed using the wick technique ${ }^{1}$ at the end of the stabilization period and for each consecutive hour of CPB.

\section{Determination of Inflammatory Markers}

Before surgery, at the end of stabilization, and after 1,2, and 3 hours of $\mathrm{CPB}$, blood samples were taken and centrifuged at $3000 \mathrm{rpm}$ for 10 minutes for cytokine analysis.

The samples were prepared for analysis in duplicates using the enzymelinked immunosorbent assay technique, as described by the manufacturer (R\&D Systems, Abingdon, UK). Total surface fluorescence was measured using an EMax Microplate Reader (Molecular Devices, Sunnyvale, Calif). The cytokine concentration was calculated with reference to standard curves: tumor necrosis factor- $\alpha$, range 23.4 to $1500 \mathrm{pg} / \mathrm{mL}$, interleukin (IL)-6, range 18.8 to $1200 \mathrm{pg} / \mathrm{mL}$, and IL-10, range 31.2 to $2000 \mathrm{pg} / \mathrm{mL}$.

\section{Procedures at Experiment End}

At the end of each experiment, the pigs were killed by an intravenous injection of $20 \mathrm{~mL}$ of saturated potassium chloride solution.

Immediately thereafter, 3 parallel tissue samples were taken simultaneously from a number of organs, processed, ${ }^{1}$ and compared with a historic control of 13 pigs killed immediately after the initiation of anesthesia.

\section{Statistical Analysis}

Statistical analysis was performed using the Statistical Package for Social Sciences, version 13.0, for Windows (SPSS Inc, Chicago, Ill). Repeated measures analysis of variance with 1 grouping variable was used to test the relationship of the outcome variables at different times. Finding a significant within-group $P$ value, a post hoc paired $t$ test was used to assess the changes at the end of CPB in relation to the preCPB baseline. If a significant between-group $P$ value was found, post hoc $t$ tests were performed to compare the 2 groups before and at the end of CPB. To compare the total tissue water content (TTW), 1-way analysis of variance was performed (GraphPad Instat, GraphPad Software Inc, La Jolla, Calif).

For the markers of general inflammation, the Friedman test was used on each group separately. If a significant overall $P$ value was found, the Wilcoxon signed rank test was used to compare the pre-CPB and 180-minute CPB values. The Mann-Whitney $U$ test was applied to compare the 180 minute CPB mean values between the 2 study groups.
A significance level of $P<.05$ was chosen. The $P$ values were adjusted according to the number of comparisons. All variables are presented as the mean \pm the standard error of the mean.

In a number of previous experiments of the FER during $\mathrm{CPB}$, a post hoc power analysis yielded a power $>90 \%$ that justified study groups of 7 animals/group $^{1}$ (StatMate, version 2.00 for Windows, GraphPad Instat, GraphPad Software Inc). Concerning the effects on FER, groups of 8 animals were considered sufficient for the detection of important changes.

\section{RESULTS}

The pigs in the PP and NP groups were comparable with respect to age $(89.4 \pm 5.5$ and $84.9 \pm 4.4$ days $)$, weight $(33.1 \pm 0.7$ and $33.4 \pm 0.7 \mathrm{~kg}$ ), and gender ( 3 male and 5 female and 4 male and 4 female, respectively). All the pigs were kept normothermic during the experiments.

\section{Laboratory Parameters, PV, NFB, and FER}

After initiating CPB, the hematocrit decreased in both study groups, with no between-group differences, although the values of the PP group tended to be slightly less than the values of the NP group (Table 1). The acid-base parameters and serum electrolyte concentrations are listed in Table 1. The respective values changed similarly, but remained essentially within the normal range in both groups, with no between-group differences (Table 1).

The serum albumin and serum protein concentration decreased in both groups, together with the COP in plasma and the $\mathrm{COP}_{\mathrm{i}}$. No between-group differences were found (Table $1)$. The albumin and protein masses remained essentially unchanged during the experiments (Table 1).

The PV of the PP and NP group before CPB was $49.33 \pm$ $1.64 \mathrm{~mL} / \mathrm{kg}$ and $49.17 \pm 3.78 \mathrm{~mL} / \mathrm{kg}$, respectively. After initiation of CPB, the PV decreased in both groups to $38.09 \pm$ $2.11 \mathrm{~mL} / \mathrm{kg}$ in the PP group and $34.66 \pm 4.37 \mathrm{~mL} / \mathrm{kg}$ in the NP group at 60 minutes. Thereafter, the PV of the PP group returned to pre-CPB levels $(49.11 \pm 3.47 \mathrm{~mL} / \mathrm{kg})$ after $180 \mathrm{~min}$ utes (Figure 1, $A$ ). However, the PV of the NP group remained contracted at $41.96 \pm 7.04 \mathrm{~mL} / \mathrm{kg}(P=.02$; Figure $1, A)$.

Figure 1, $B$ displays the NFB values at 30 -minute intervals before and during CPB. No within- or between-group differences were obtained. The values of the PP group, however, tended to stay higher than the values of the NP group throughout the experiments.

The FER was $0.22 \pm 0.04 \mathrm{~mL} / \mathrm{kg} / \mathrm{min}$ and $0.26 \pm 0.04$ $\mathrm{mL} / \mathrm{kg} / \mathrm{min}$ in the PP and NP groups, respectively, before $\mathrm{CPB}$ and changed similarly during $\mathrm{CPB}$, with no betweenor within-group differences. The FER values at 30-minute intervals before and during 180 minutes of CPB are presented in Figure 1, $C$. Despite the lack of significant withinor between-group differences, the FER of the PP group tended to stay slightly higher than the values of the NP group at all intervals (Figure 1,C). 
TABLE 1. Laboratory parameters

\begin{tabular}{|c|c|c|c|c|c|c|c|c|}
\hline \multirow[b]{2}{*}{ Parameter } & \multirow[b]{2}{*}{ Before CPB } & \multicolumn{6}{|c|}{ CPB (min) } & \multirow[b]{2}{*}{$P_{\mathrm{w}}$ value } \\
\hline & & 30 & 60 & 90 & 120 & 150 & 180 & \\
\hline \multicolumn{9}{|l|}{ Hematocrit (\%) } \\
\hline PP & $28.1 \pm 1.1$ & $21.4 \pm 1.1$ & $22.1 \pm 1.0$ & $21.5 \pm 0.9$ & $20.9 \pm 0.7$ & $20.1 \pm 0.9$ & $19.3 \pm 1.0$ & .000 \\
\hline NP & $28.5 \pm 0.9$ & $22.5 \pm 0.7$ & $22.9 \pm 0.7$ & $22.5 \pm 0.7$ & $22.1 \pm 0.5$ & $22.3 \pm 0.5$ & $21.3 \pm 0.4$ & .000 \\
\hline \multicolumn{9}{|l|}{$\mathrm{pH}$} \\
\hline PP & $7.53 \pm 0.02$ & $7.45 \pm 0.05$ & $7.44 \pm 0.05$ & $7.44 \pm 0.03$ & $7.45 \pm 0.03$ & $7.43 \pm 0.04$ & $7.44 \pm 0.08$ & .035 \\
\hline NP & $7.54 \pm 0.02$ & $7.48 \pm 0.01$ & $7.47 \pm 0.01$ & $7.45 \pm 0.01$ & $7.45 \pm 0.02$ & $7.46 \pm 0.02$ & $7.49 \pm 0.02$ & NS \\
\hline \multicolumn{9}{|l|}{$\mathrm{pCO}_{2}(\mathrm{kPa})$} \\
\hline PP & $4.8 \pm 0.1$ & $5.8 \pm 0.1$ & $5.8 \pm 0.1$ & $5.6 \pm 0.1$ & $5.6 \pm 0.1$ & $5.7 \pm 0.1$ & $5.7 \pm 0.1$ & NS \\
\hline NP & $4.9 \pm 0.2$ & $5.7 \pm 0.1$ & $5.9 \pm 0.1$ & $5.8 \pm 0.1$ & $5.7 \pm 0.1$ & $5.6 \pm 0.1$ & $5.5 \pm 0.1$ & NS \\
\hline \multicolumn{9}{|l|}{$\mathrm{BE}(\mathrm{mmol} / \mathrm{L})$} \\
\hline $\mathrm{PP}$ & $6.3 \pm 0.6$ & $6.1 \pm 1.3$ & $6.4 \pm 1.4$ & $4.8 \pm 1.1$ & $5.4 \pm 1.2$ & $5.1 \pm 1.4$ & $5.3 \pm 1.9$ & NS \\
\hline NP & $7.8 \pm 0.6$ & $9.1 \pm 0.5$ & $9.3 \pm 0.8$ & $6.5 \pm 0.8$ & $6.3 \pm 1.3$ & $6.4 \pm 1.2$ & $7.6 \pm 1.5$ & NS \\
\hline \multicolumn{9}{|l|}{ Serum sodium $(\mathrm{mmol} / \mathrm{L})$} \\
\hline PP & $138.8 \pm 0.5$ & $138.8 \pm 0.5$ & $138.6 \pm 0.4$ & $139.0 \pm 0.6$ & $138.6 \pm 0.4$ & $138.1 \pm 0.4$ & $138.5 \pm 0.5$ & NS \\
\hline NP & $139.1 \pm 0.6$ & $139.3 \pm 0.5$ & $139.1 \pm 0.5$ & $139.1 \pm 0.5$ & $139.4 \pm 0.2$ & $139.5 \pm 0.3$ & $139.4 \pm 0.2$ & NS \\
\hline \multicolumn{9}{|c|}{ Serum potassium $(\mathrm{mmol} / \mathrm{L})$} \\
\hline PP & $4.3 \pm 0.1$ & $4.4 \pm 0.1$ & $4.4 \pm 0.1$ & $4.6 \pm 0.1$ & $4.8 \pm 0.1$ & $5.3 \pm 0.2$ & $5.8 \pm 0.2$ & .002 \\
\hline NP & $4.3 \pm 0.1$ & $4.3 \pm 0.1$ & $4.1 \pm 0.1$ & $4.4 \pm 0.1$ & $4.7 \pm 0.1$ & $4.9 \pm 0.2$ & $5.3 \pm 0.1$ & .000 \\
\hline \multicolumn{9}{|c|}{ Serum chloride $(\mathrm{mmol} / \mathrm{L})$} \\
\hline $\mathrm{PP}$ & $98.6 \pm 1.0$ & $102.1 \pm 1.0$ & $101.5 \pm 0.9$ & $102.3 \pm 1.1$ & $102.1 \pm 0.9$ & $102.4 \pm 1.0$ & $102.8 \pm 1.1$ & .002 \\
\hline NP & $97.1 \pm 0.6$ & $101.1 \pm 0.7$ & $100.8 \pm 0.5$ & $100.9 \pm 0.3$ & $100.8 \pm 0.6$ & $101.3 \pm 0.5$ & $102.0 \pm 0.5$ & .000 \\
\hline \multicolumn{9}{|c|}{ Serum osmolality (mosmol/kg) } \\
\hline $\mathrm{PP}$ & $285.3 \pm 1.3$ & $285.8 \pm 1.0$ & $287.0 \pm 1.2$ & $285.3 \pm 0.8$ & $285.1 \pm 1.0$ & $285.9 \pm 1.5$ & $285.5 \pm 1.5$ & NS \\
\hline NP & $287.1 \pm 0.9$ & $285.9 \pm 1.3$ & $286.5 \pm 1.3$ & $287.0 \pm 1.3$ & $287.4 \pm 1.0$ & $287.8 \pm 0.9$ & $288.1 \pm 1.2$ & NS \\
\hline \multicolumn{9}{|l|}{ Serum albumin $(\mathrm{g} / \mathrm{L})$} \\
\hline PP & $28.6 \pm 1.1$ & $20.0 \pm 1.1$ & $20.5 \pm 1.2$ & $20.3 \pm 1.1$ & $19.9 \pm 1.1$ & $18.8 \pm 1.1$ & $18.4 \pm 1.2$ & .000 \\
\hline NP & $28.7 \pm 0.6$ & $20.4 \pm 0.4$ & $20.5 \pm 0.6$ & $19.9 \pm 0.6$ & $20.3 \pm 0.7$ & $19.9 \pm 0.5$ & $19.1 \pm 0.6$ & .000 \\
\hline \multicolumn{9}{|l|}{ Serum protein $(g / L)$} \\
\hline $\mathrm{PP}$ & $47.0 \pm 1.5$ & $32.4 \pm 1.3$ & $33.4 \pm 1.6$ & $32.8 \pm 1.4$ & $32.3 \pm 1.2$ & $30.9 \pm 1.5$ & $29.8 \pm 1.7$ & .000 \\
\hline NP & $46.1 \pm 0.9$ & $33.8 \pm 0.4$ & $33.8 \pm 0.4$ & $33.3 \pm 0.6$ & $33.0 \pm 0.8$ & $32.9 \pm 0.8$ & $31.8 \pm 0.9$ & .000 \\
\hline \multicolumn{9}{|l|}{ Albumin mass (g) } \\
\hline PP & $48.3 \pm 3.4$ & $48.6 \pm 4.2$ & $47.4 \pm 4.3$ & $48.0 \pm 4.1$ & $48.8 \pm 4.2$ & $48.1 \pm 3.8$ & $49.3 \pm 4.1$ & NS \\
\hline NP & $46.6 \pm 3.1$ & $46.8 \pm 3.6$ & $45.9 \pm 3.6$ & $45.3 \pm 3.8$ & $46.9 \pm 3.9$ & $45.5 \pm 3.4$ & $46.0 \pm 3.2$ & NS \\
\hline \multicolumn{9}{|l|}{ Protein mass (g) } \\
\hline PP & $79.7 \pm 5.9$ & $78.6 \pm 6.3$ & $77.4 \pm 7.0$ & $78.1 \pm 6.8$ & $79.3 \pm 6.1$ & $79.2 \pm 6.0$ & $80.1 \pm 6.5$ & NS \\
\hline NP & $77.4 \pm 4.9$ & $77.5 \pm 5.7$ & $75.7 \pm 5.6$ & $75.6 \pm 5.6$ & $76.4 \pm 5.9$ & $75.4 \pm 5.8$ & $76.3 \pm 5.2$ & NS \\
\hline \multicolumn{9}{|l|}{$\mathrm{COP}_{\mathrm{p}}(\mathrm{mm} \mathrm{Hg})$} \\
\hline PP* & $13.7 \pm 0.4$ & $8.5 \pm 0.5$ & $9.0 \pm 0.5$ & $9.0 \pm 0.4$ & $8.5 \pm 0.4$ & $8.7 \pm 0.4$ & $8.1 \pm 0.5$ & .000 \\
\hline $\mathrm{NP}^{*}$ & $13.7 \pm 0.3$ & $9.3 \pm 0.2$ & $9.3 \pm 0.3$ & $9.3 \pm 0.3$ & $9.2 \pm 0.4$ & $9.3 \pm 0.3$ & $8.5 \pm 0.4$ & .000 \\
\hline \multicolumn{9}{|l|}{$\mathrm{COP}_{\mathrm{i}}(\mathrm{mm} \mathrm{Hg})$} \\
\hline PP* & $7.5 \pm 0.7$ & & $6.0 \pm 0.5$ & & $5.7 \pm 0.3$ & & $5.3 \pm 0.3$ & .003 \\
\hline NP & $7.3 \pm 0.4$ & & $6.3 \pm 0.5$ & & $5.9 \pm 0.5$ & & $5.6 \pm 0.3$ & .004 \\
\hline
\end{tabular}

Data are presented as the mean \pm standard error of the mean. $P$ values for between-group difference at 180 minutes of CPB were not statistically significant. $C P B$, Cardiopulmonary bypass; $P_{w}$ value, $P$ value for within-group differences at 180 minutes compared with before CPB; $P P$, pulsatile perfusion (group); $N P$, nonpulsatile perfusion (group); $N S$, not significant; $\mathrm{PCO}_{2}$, partial pressure of carbon dioxide; $B E$, base excess; $C O P_{p}$, colloid osmotic pressure in plasma; $C O P_{i}$, colloid osmotic pressure in interstitial fluid. *n $=7$.

\section{Hemodynamic Parameters, EEP, and SHE}

The mean arterial pressure (MAP) before CPB was 61.1 $\pm 5.4 \mathrm{~mm} \mathrm{Hg}$ and $64.5 \pm 3.1 \mathrm{~mm} \mathrm{Hg}$ in the PP and $\mathrm{NP}$ groups, respectively. After starting $\mathrm{CPB}$, the MAP increased in the PP group, but a decrease was seen in the NP group (Table 2). At 180 minutes of CPB, the MAP of the PP group was $70.9 \pm 2.7 \mathrm{~mm} \mathrm{Hg}$ compared with 55.9 $\pm 2.7 \mathrm{~mm} \mathrm{Hg}$ in the NP group (between-group difference, $P=.004$; Table 2).

The CVP in the right femoral vein remained unchanged from the pre-CPB values in both study groups throughout the experiments (Table 2). In contrast, the CVP of the right atrium decreased significantly in the NP group on the start of $\mathrm{CPB}$ from $5.0 \pm 0.8 \mathrm{~mm} \mathrm{Hg}$ to $1.4 \pm 0.5 \mathrm{~mm} \mathrm{Hg}$ at 

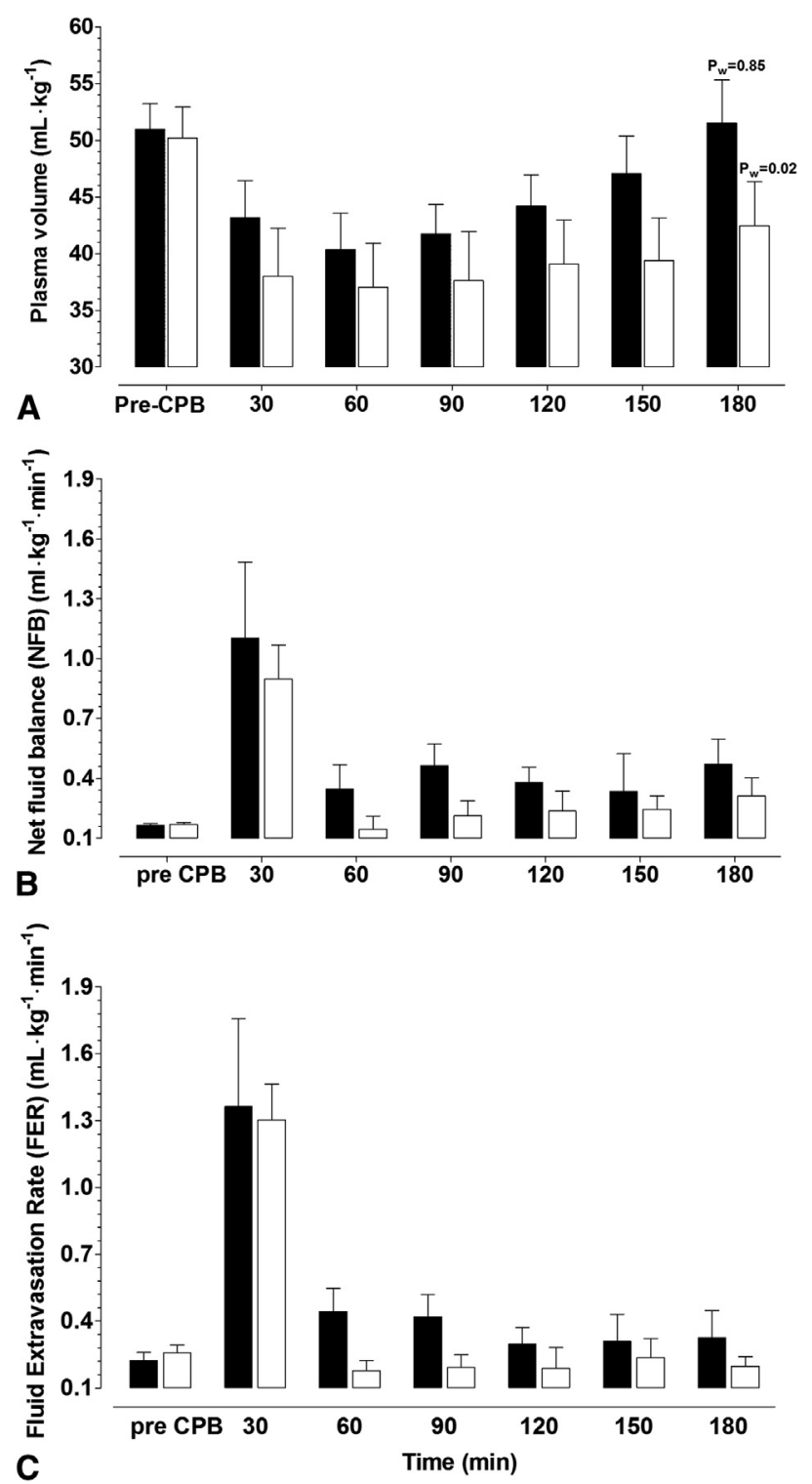

FIGURE 1. A, The plasma volume before and during 180 minutes of cardiopulmonary bypass $(C P B)$. B, Net fluid balance before and during 180 minutes of CPB. C, Fluid extravasation rate before and during 180 minutes of CPB. Black columns, pulsatile flow; white columns, nonpulsatile flow. Data are presented as mean \pm standard error of the mean.

180 minutes $(P=.002)$. In the PP group, the decrease in CVP approached significance $(P=.08)$. No betweengroup differences were observed in atrial CVP, although the values of the PP group tended to stay slightly higher than the values of the NP group at all intervals (Table 2).

The EEP and SHE were calculated before and during CPB. Before the start of CPB, the EEP was $71.32 \pm 3.52 \mathrm{~mm} \mathrm{Hg}$ in the PP group and $73.79 \pm 2.28 \mathrm{~mm} \mathrm{Hg}$ in the NP group $(P=.57)$. The corresponding values for SHE were $12,412.0 \pm 1962.6 \mathrm{erg} / \mathrm{cm}^{3}$ and $8241.2 \pm 746.5 \mathrm{erg} / \mathrm{cm}^{3}$ $(P=.07)$. After the start of CPB, the EEP and SHE remained stable during the bypass period. The EEP and SHE of the PP group was typically $85.9 \pm 16.2 \mathrm{~mm} \mathrm{Hg}$ and $7660.6 \pm 2527.2$ $\mathrm{erg} / \mathrm{cm}^{3}$, respectively. No differences were present in the preCPB SHE and during-CPB SHE in the PP group $(P=.16)$. The corresponding values for EEP and SHE in the NP group were $57.9 \pm 7.2 \mathrm{~mm} \mathrm{Hg}$ and $11.9 \pm 11.2 \mathrm{erg} / \mathrm{cm}^{3}$.

\section{Cytokines}

The tumor necrosis factor- $\alpha$, IL- 6 , and IL-10 values are listed in Table 3. The tumor necrosis factor- $\alpha$ values at 180 minutes were similar to those before CPB. The IL-6 levels were significantly elevated in both study groups $(P=.012)$ at 180 minutes of CPB compared with before CPB. No between-group differences were obtained. Concerning IL-10, no within- or between-group differences were present.

\section{Total Tissue Water Content}

The TTW of the respective organs are listed in Table 4. The use of CPB resulted in an increase in the TTW in most organs within both groups compared with the historic control animals that had never undergone CPB. Between-group differences (PP vs NP group) were, however, absent for all organs.

\section{DISCUSSION}

The use of CPB during open heart surgery has been hampered by undesirable effects that can interfere with fluid homeostasis, result in edema formation, and, occasionally, interfere with organ function. Restrictive perioperative fluid therapy has been associated with improved postoperative organ function, a lower incidence of postoperative complications, and, probably, shorter hospital stays ${ }^{21}$ in contrast to a highly positive intraoperative fluid balance $(>5 \mathrm{~L})$, which has been associated with adverse outcomes. ${ }^{22}$

The perfusion mode and flow pattern are among a number of factors that can affect fluid accumulation during CPB., ${ }^{9,23}$ Considerable evidence in favor of pulsatile CPB flow has accumulated from studies of neonates, infants, small children, and animals, ${ }^{23}$ in which the damaging effects on erythrocytes and thrombocytes were similar with pulsatile and nonpulsatile perfusion. PP lowers pulmonary vascular resistance, improves microcirculation and metabolism by its higher hemodynamic energy, and contributes to improve pulmonary function and reduce the general inflammatory reaction observed during $\mathrm{CPB} .^{23}$ Thus, fluid and protein extravasation and edema formation after CPB could be inhibited.

A number of recent studies have focused on the beneficial effects of preoperative IABP deployment in high-risk groups undergoing coronary artery bypass grafting, ${ }^{11,12}$ but also on the continuation of IABP-PP during CPB. ${ }^{14,16,17}$ Its use during CPB and cardioplegic arrest has preserved lung function in patients with obstructive pulmonary disease ${ }^{17}$ and has contributed to renal, hepatic, and pancreatic preservation. ${ }^{13}$ Finally, regional and global 
TABLE 2. Hemodynamic parameters

\begin{tabular}{|c|c|c|c|c|c|c|c|c|c|}
\hline \multirow[b]{2}{*}{ Parameter } & \multirow[b]{2}{*}{ Before CPB } & \multicolumn{6}{|c|}{ CPB (min) } & \multirow[b]{2}{*}{$P_{\mathrm{w}}$ value } & \multirow[b]{2}{*}{$P_{\mathrm{b}}$ value } \\
\hline & & 30 & 60 & 90 & 120 & 150 & 180 & & \\
\hline \multicolumn{10}{|l|}{ MAP (mm Hg) } \\
\hline PP & $61.1 \pm 5.4$ & $80.8 \pm 6.7$ & $80.0 \pm 7.4$ & $81.1 \pm 7.4$ & $79.9 \pm 5.0$ & $77.0 \pm 3.2$ & $70.9 \pm 2.7$ & NS & .004 \\
\hline NP & $64.5 \pm 3.1$ & $54.1 \pm 2.6$ & $53.7 \pm 2.8$ & $52.4 \pm 2.2$ & $57.4 \pm 2.4$ & $55.7 \pm 2.3$ & $55.9 \pm 2.7$ & NS & \\
\hline \multicolumn{10}{|c|}{$\mathrm{CVP}$, right atrium $(\mathrm{mm} \mathrm{Hg})$} \\
\hline $\mathrm{PP}$ & $4.4 \pm 1.1$ & $2.3 \pm 0.9$ & $2.4 \pm 0.7$ & $2.4 \pm 0.8$ & $2.0 \pm 0.9$ & $2.0 \pm 1.1$ & $2.5 \pm 1.1$ & NS & NS \\
\hline NP & $5.0 \pm 0.8$ & $0.9 \pm 0.4$ & $0.7 \pm 0.4$ & $0.6 \pm 0.4$ & $1.3 \pm 0.5$ & $0.7 \pm 0.5$ & $1.4 \pm 0.5$ & .002 & \\
\hline \multicolumn{10}{|c|}{$\begin{array}{l}\text { CVP, right femoral vein } \\
(\mathrm{mm} \mathrm{Hg})\end{array}$} \\
\hline PP & $5.4 \pm 0.9$ & $6.1 \pm 0.9$ & $6.1 \pm 0.9$ & $6.1 \pm 0.9$ & $6.5 \pm 0.9$ & $6.6 \pm 1.1$ & $6.9 \pm 1.2$ & NS & NS \\
\hline NP & $5.8 \pm 1.2$ & $5.4 \pm 0.9$ & $5.6 \pm 1.0$ & $5.7 \pm 1.0$ & $5.6 \pm 1.0$ & $5.9 \pm 1.2$ & $6.0 \pm 1.1$ & NS & \\
\hline \multicolumn{10}{|l|}{ SVRI (dyne $/ \mathrm{s} / \mathrm{cm}^{5} / \mathrm{m}^{2}$ ) } \\
\hline $\mathrm{PP}$ & $1555 \pm 143$ & $1789 \pm 156$ & $1769 \pm 177$ & $1794 \pm 173$ & $1779 \pm 126$ & $1706 \pm 79$ & $1555 \pm 72$ & NS & .004 \\
\hline NP & $1429 \pm 139$ & $1205 \pm 53$ & $1197 \pm 58$ & $1171 \pm 46$ & $1267 \pm 44$ & $1243 \pm 50$ & $1230 \pm 62$ & NS & \\
\hline \multicolumn{10}{|l|}{$\mathrm{CO}(\mathrm{L} / \mathrm{min})$} \\
\hline PP & $2.8 \pm 0.3$ & $3.3 \pm 0.1$ & $3.3 \pm 0.1$ & $3.3 \pm 0.1$ & $3.3 \pm 0.1$ & $3.3 \pm 0.1$ & $3.3 \pm 0.1$ & NS & NS \\
\hline NP & $3.3 \pm 0.3$ & $3.3 \pm 0.1$ & $3.3 \pm 0.1$ & $3.3 \pm 0.1$ & $3.3 \pm 0.1$ & $3.3 \pm 0.1$ & $3.3 \pm 0.1$ & NS & \\
\hline
\end{tabular}

Data are presented as the mean \pm standard error of the mean. $P_{b}$ value, $P$ value for between-group differences at 180 minutes of CPB; MAP, mean arterial pressure; $C V P$, central venous pressure; $S V R I$, body surface area indexed systemic vascular resistance; $C O$, cardiac output; $C P B$, cardiopulmonary bypass; $P_{w}$ value, $P$ value for within-group differences at 180 minutes compared with before CPB; $P P$, pulsatile perfusion (group); $N P$, nonpulsatile perfusion (group); $N S$, not significant.

perfusion during CPB and throughout the early postoperative period has been shown to be improved. ${ }^{14}$

To date, no studies have focused on microvascular fluid exchange during CPB with PP by the concomitant intraoperative use of IABP. In the present study, we addressed this topic, with special attention to the changes in PV, NFB, and transcapillary fluid exchange with the IABP switched to an automatic 80 beats/min mode immediately at the initiation of CPB (PP group) or switched off during the experiments (NP group).

Our main findings are briefly listed below and will be discussed in the order presented:

IABP resulted in PP with SHE values during maintained $\mathrm{CPB}$

Between-group differences were obtained for the MAP and body surface area indexed systemic vascular resistance, with higher values in the PP group
No between-group differences were present in NFB and FER, although the PP group values tended to be slightly elevated compared with those of the NP group

The PV decreased in both groups with initiation of CPB; in the PP group, the PV returned to its pre-CPB level but remained contracted in the NP group

The right atrial CVP decreased significantly in the NP group, but not in the PP group; although betweengroup differences were absent, the values of the PP group tended to be slightly elevated compared with those of the NP group

$\mathrm{COP}$ in plasma and $\mathrm{COP}_{\mathrm{i}}$ decreased significantly in both groups during $\mathrm{CPB}$

CPB induced a similar inflammatory response in both groups

The TTW changed similarly in both study groups

TABLE 3. Markers of general inflammation

\begin{tabular}{|c|c|c|c|c|c|c|}
\hline \multirow[b]{2}{*}{ Parameter } & \multirow[b]{2}{*}{ Preoperatively } & \multirow[b]{2}{*}{ Before CPB } & \multicolumn{3}{|c|}{ CPB (min) } & \multirow[b]{2}{*}{$P_{\mathrm{w}}$ value } \\
\hline & & & 60 & 120 & 180 & \\
\hline \multicolumn{7}{|c|}{$\mathrm{TNF}-\alpha(\mathrm{pg} / \mathrm{mL})$} \\
\hline $\mathrm{PP}$ & $73.6 \pm 7.8$ & $311.8 \pm 84.2$ & $966.8 \pm 448.9$ & $649.6 \pm 205.0$ & $472.3 \pm 106.5$ & .263 \\
\hline NP & $63.0 \pm 4.7$ & $523.3 \pm 274.0$ & $1761.8 \pm 578.5$ & $881.6 \pm 225.6$ & $500.0 \pm 111.8$ & .401 \\
\hline \multicolumn{7}{|l|}{ IL-6 (pg/mL) } \\
\hline PP & $7.9 \pm 3.7$ & $75.8 \pm 35.3$ & $831.3 \pm 159.8$ & $1491.0 \pm 229.4$ & $1757.4 \pm 241.8$ & .012 \\
\hline NP & $4.8 \pm 1.9$ & $65.3 \pm 13.5$ & $1202.8 \pm 392.7$ & $1678.8 \pm 483.4$ & $1246.4 \pm 327.6$ & .012 \\
\hline \multicolumn{7}{|l|}{ IL-10 (pg/mL) } \\
\hline PP & $49.6 \pm 9.0$ & $47.0 \pm 2.3$ & $45.4 \pm 3.3$ & $40.9 \pm 5.9$ & $44.9 \pm 6.7$ & NS \\
\hline NP & $30.3 \pm 2.1$ & $48.9 \pm 6.6$ & $57.0 \pm 8.8$ & $49.4 \pm 6.9$ & $45.6 \pm 4.0$ & NS \\
\hline
\end{tabular}

Data are presented as the mean \pm standard error of the mean. $P$ values for between-group difference at 180 minutes of CPB were not statistically significant. TNF, Tumor necrosis factor; $I L$, interleukin; $C P B$, cardiopulmonary bypass; $P_{w}$ value, $P$ value for within-group differences at 180 minutes compared with before $C P B ; P P$, pulsatile perfusion (group); $N P$, nonpulsatile perfusion (group); $N S$, not significant. 
TABLE 4. Total tissue water content (g/g of dry weight) after CPB

\begin{tabular}{|c|c|c|c|c|c|}
\hline Tissue & $C$ group $(n=13)$ & $P P \operatorname{group}(n=8)$ & $P$ value (PP vs C) & NP group $(n=8)$ & $P$ value (NP vs C) \\
\hline Right myocardium & $4.17 \pm 0.02$ & $4.50 \pm 0.10$ & $<.001$ & $4.36 \pm 0.04$ & $<.05$ \\
\hline Left myocardium & $4.00 \pm 0.04$ & $4.12 \pm 0.09$ & NS & $4.21 \pm 0.06$ & NS \\
\hline Lung & $4.16 \pm 0.07$ & $6.32 \pm 0.40$ & $<.001$ & $6.87 \pm 0.74$ & $<.001$ \\
\hline Liver & $2.89 \pm 0.05$ & $3.37 \pm 0.23$ & $<.05$ & $3.19 \pm 0.04$ & NS \\
\hline Left kidney & $4.48 \pm 0.07$ & $4.69 \pm 0.14$ & NS & $5.10 \pm 0.24$ & $<.05$ \\
\hline Right kidney & $4.56 \pm 0.08$ & $4.82 \pm 0.13$ & NS & $5.01 \pm 0.21$ & NS \\
\hline \multicolumn{6}{|l|}{ Stomach } \\
\hline Muscularis & $4.03 \pm 0.09$ & $5.41 \pm 0.19$ & $<.001$ & $5.52 \pm 0.35$ & $<.001$ \\
\hline Mucosa & $4.41 \pm 0.08$ & $4.65 \pm 0.18$ & NS & $4.69 \pm 0.28$ & NS \\
\hline Pancreas & $3.40 \pm 0.10$ & $4.03 \pm 0.13$ & $<.01$ & $4.40 \pm 0.21$ & $<.001$ \\
\hline \multicolumn{6}{|l|}{ Ileum } \\
\hline Muscularis & $3.65 \pm 0.18$ & $4.62 \pm 0.25$ & $<.01$ & $4.88 \pm 0.10$ & $<.001$ \\
\hline Mucosa & $4.72 \pm 0.05$ & $5.56 \pm 0.34$ & $<.01$ & $5.55 \pm 0.10$ & $<.01$ \\
\hline Colon & $3.85 \pm 0.19$ & $4.98 \pm 0.32$ & $<.05$ & $5.33 \pm 0.47$ & $<.01$ \\
\hline Skeletal muscle & $3.43 \pm 0.04$ & $3.87 \pm 0.11$ & $<.05$ & $3.95 \pm 0.18$ & $<.01$ \\
\hline Skin & $1.93 \pm 0.07$ & $2.30 \pm 0.17$ & NS & $2.32 \pm 0.19$ & NS \\
\hline Brain & $3.74 \pm 0.09$ & $4.38 \pm 0.14$ & $<.001$ & $4.42 \pm 0.09$ & $<.001$ \\
\hline
\end{tabular}

Data are presented as mean \pm SEM. All $P$ values for PP versus NP groups were not statistically significant. $C$ group, Control group (never underwent $C P B$ ); $C P B$, cardiopulmonary bypass; $P P$, pulsatile perfusion (group); $N P$, nonpulsatile perfusion (group); $N S$, not significant.

\section{EEP and SHE}

It has been recommended that one precisely quantify the arterial pressure and waveform to produce useful hemodynamic comparisons between different perfusion modes and studies. ${ }^{19}$ The EEP and SHE were, therefore, calculated. The EEP and SHE values remained stable during CPB.

The clear differences in SHE in the 2 study groups during CPB have demonstrated the ability of IABP to generate a pulsatile flow. Additionally, the similar values of SHE before and during CPB (PP group) demonstrated near physiologic pulsatility in terms of SHE.

\section{MAP, Cardiac Output, and Systemic Vascular Resistance}

Sympathetic vasomotor innervation can influence microvascular fluid shifts by the effects on the pre- to postcapillary resistance ratio, resulting in a decrease in the Pc with lowered fluid filtration. The cardiac output was held constant in the present study. Therefore, according to the MAP $=$ cardiac output $\times$ systemic vascular resistance (SVR) formula, the higher MAP of the PP group should normally reflect an increase in the SVR, with lowered Pc values and lowered fluid filtration, opposite the trends observed.

The calculated values of SVR and our interpretation of the results were complicated because the distribution of cardiac output relative to the position of the IABP balloon remains unknown, because the balloon might represent a partial obstruction to blood flow. Therefore, different pressures and SVRs above and beneath the balloon might have been present, resulting in different conditions for fluid shifts and fluid loading and unloading.

\section{NFB, FER, COP, CVP, PV, and TTW}

No significant between-group differences were obtained for the NFB and FER when analyzing every 30-minute interval of the experiments. Nor was any between-group differences for $\mathrm{COP}_{\mathrm{i}}$ observed. In both study groups, a decrease in $\mathrm{COP}_{\mathrm{i}}$ was found, reflecting a continuous shift of fluid from the circulation to the interstitial space throughout the experiments. This conclusion was also confirmed by the significant increase in the TTW in a number of organs of the PP and NP groups compared with the historic control values obtained from animals that had never undergone CPB.

The Pc is influenced by the CVP according to the equation: $\mathrm{Pc}=\mathrm{CVP}+\mathrm{F} \times \mathrm{Rv}$, where $\mathrm{F}$ is blood flow and $\mathrm{Rv}$ is the venous resistance. In the present study, a trend was seen for higher CVP values in the PP group, probably contributing to an increase in Pc, affecting the FER trend observed during PP.

The increase in the PV during CPB in the PP group could also have been related to the CVP. Venous compliance is about 50 times that of arterial compliance, and even small changes in venous pressure can lead to extensive volume accumulation. ${ }^{4}$

PP is well known to enhance arterial wall stress, related to flow speed acceleration and turbulence, and results in an increase in nitric oxide production. ${ }^{15}$ Nitric oxide-induced vasodilatation could also have contributed to the elevation of PV back to pre-CPB levels.

The trend toward elevated CVP values in the PP group compared with the NP group is difficult to interpret, because a fixed height differences between the machine reservoir and the site of venous drainage (the right atrium) was constant and similar in both groups during $\mathrm{CPB}$. Free 
drainage was also followed by visual inspection and by continuously controlling the correct position of the venous return cannula in the right atrium. To what extent intraaortic balloon pumping might have contributed to the slight increase in the CVP in the PP group is unclear. In a previous report, ${ }^{24}$ a hemodynamic examination was performed in cardiac arrest victims with an IABP used during resuscitation. Descending aortic occlusion by the IABP was associated with a marked increase in the CVP to values greater than that observed in the present study. ${ }^{24}$

\section{Inflammation Markers}

Cardiac surgery involving CPB is well known to provoke an inflammatory reaction related to the contact of blood with the foreign surfaces of the CPB circuit, tissue injury, ischemia-reperfusion injury, and the release of endotoxins, ${ }^{7}$ contributing to an increase in vascular permeability to water and proteins. In the present study, the pattern of proinflammatory markers was similar in both groups and essentially in line with the study by Onorati et $\mathrm{al}^{25}$ in which inflammation was identical whether the perfusion was pulsatile or nonpulsatile.

\section{CONCLUSIONS}

In the present study, we used a nonpulsatile pump and added IABP as a surrogate to achieve pulsatile flow during $\mathrm{CPB}$. However, the microvascular fluid extravasation was similar, whether the CPB flow was pulsatile or nonpulsatile.

A slight, insignificant, trend toward greater FERs during PP might have been related to aortic occlusion by the IABP and associated changes in the CVP. Compared with the control animals that had never undergone $\mathrm{CPB}$, both IABP-induced pulsatile and nonpulsatile CPB perfusion contributed similarly to fluid loading and edema generation in this experimental setup.

The Board of the University of Bergen Faculty of Medicine has authorized the "Locus for Circulatory Research" as an officially recognized research group within the faculty. We greatly acknowledge this support. Steinar Lundemoen is a research fellow supported by the Department for Heart Disease at the Haukeland University Hospital and the Grieg Foundation, Bergen, Norway. The technical assistance of Lill Andreasen, Cato Johnsen, and Gry Hilde Nilsen is greatly appreciated.

\section{References}

1. Farstad M, Heltne JK, Rynning SE, Onarheim H, Mongstad A, Eliassen F, et al. Can the use of methylprednisolone, vitamin $\mathrm{C}$, or alpha-trinositol prevent cold-induced fluid extravasation during cardiopulmonary bypass in piglets? J Thorac Cardiovasc Surg. 2004;127:525-34.

2. Kvalheim VL, Farstad M, Steien E, Mongstad A, Borge BA, Kvitting PM, et al. Infusion of hypertonic saline/starch during cardiopulmonary bypass reduces fluid overload and may impact cardiac function. Acta Anaesthesiol Scand. 2010;54:485-93.
3. Farstad M, Haugen O, Rynning SE, Onarheim H, Husby P. Fluid shift is moderate and short-lived during acute crystalloid hemodilution and normothermic cardiopulmonary bypass in piglets. Acta Anaesthesiol Scand. 2005;49:949-55.

4. Zhang JX, Wolf MB. Effect of cold on microvascular fluid movement in the cat limb. J Appl Physiol. 1991;71:703-8.

5. Paparelli D, Yau TM, Young E. Cardiopulmonary bypass induced inflammation: pathophysiology and treatment. An update. Eur J Cardiothorac Surg. 2002;21: 232-44.

6. Caputo M, Bays S, Rogers CA, Pawade A, Parry AJ, Suleiman S, et al. Randomized comparison between normothermic and hypothermic cardiopulmonary bypass in pediatric open-heart surgery. Ann Thorac Surg. 2005;80:982-8.

7. Haugen O, Farstad M, Kvalheim V, Bøe O, Husby P. Elevated flow rate during cardiopulmonary bypass is associated with fluid accumulation. J Thorac Cardiovasc Surg. 2007;134:587-93.

8. Connolly CM, Kramer GC, Hahn RG, Chaisson NF, Svensen CH, Kirschner RA, et al. Isoflurane but not mechanical ventilation promotes extravascular fluid accumulation during crystalloid volume loading. Anesthesiology. 2003;98:670-81.

9. Hauge A, Nicolaysen G. The importance of flow pulsatility for the rate of transvascular fluid filtration in lungs. J Physiol. 1979;290:569-76.

10. Wilkens H, Regelson W, Hoffmeister FS. The physiologic importance of pulsatile blood flow. N Eng J Med. 1962;267:443-6.

11. Christenson JT, Simonet F, Badel P, Schmuziger M. Optimal timing of preoperative intraaortic balloon pump support in high-risk coronary patients. Ann Thorac Surg. 1999;68:934-9.

12. Lavana JD, Fraser JF, Smith SE, Drake L, Tesar P, Mullany DV. Influence of timing of intraaortic balloon placement in cardiac surgical patients. J Thorac Cardiovasc Surg. 2010;140:80-5.

13. Onorati F, Cristodoro L, Mastroroberto P, di Virgilio A, Esposito A, Bilotta M, et al. Should we discontinue intraaortic balloon during cardioplegic arrest? Splanchnic function results of a prospective randomized trial. Ann Thorac Surg. 2005;80:2221-8

14. Onorati F, Santarpino G, Rubino AS, Caroleo S, Dardano A, Scalas C, et al. Body perfusion during adult cardiopulmonary bypass is improved by pulsatile flow with intra-aortic balloon pump. Int J Artif Organs. 2009;32:50-61.

15. Lanzarone E, Gelmini F, Tessari M, Menon T, Suzuki H, Carini M, et al. Preservation of endothelium nitric oxide release by pulsatile flow cardiopulmonary bypass when compared with continuous flow. Artif Organs. 2009;33:926-34.

16. Onorati F, Esposito A, Comi MC, Impiombato B, Christodoro L, Mastroroberto $\mathrm{P}$, et al. Intra-aortic balloon pump-induced pulsatile flow reduces coagulative and fibrinolytic response to cardiopulmonary bypass. Artif Organs. 2008;32:433-41.

17. Onorati F, Christodoro L, Bilotta M, Impiombato B, Pezzo F, Mastroroberto P, et al. Intraaortic balloon pumping during cardioplegic arrest preserves lung function in patients with chronic obstructive pulmonary disease. Ann Thorac Surg. 2006;82:35-43.

18. Husby P, Heltne JK, Koller ME, Birkeland S, Westby J, Fosse R, et al. Midazolam-fentanyl-isoflurane anaesthesia is suitable for haemodynamic and fluid balance studies in pigs. Lab Animal. 1998;32:316-23.

19. Undar A. The ABCs of research on pulsatile versus nonpulsatile perfusion during cardiopulmonary bypass. Med Sci Monit. 2002;8:ED21-4.

20. Heltne JK, Farstad M, Lund T, Koller ME, Matre K, Rynning SE, et al. Determination of plasma volume in anesthetized piglets using the carbon monoxide (CO) method. Lab Animal. 2002;36:344-50.

21. Toraman F, Evrenkaya S, Yuse M, Turek O, Aksoy N, Karabulut H, et al. Highly positive intraoperative fluid balance during cardiac surgery is associated with adverse outcome. Perfusion. 2004;19:85-91.

22. Morin JF, Mistry B, Langlois Y, Ma F, Chamoun P, Holcroft C. Fluid overload after coronary artery bypass grafting surgery increases the incidence of postoperative complications. WJCS. 2011;1:18-23.

23. Ji B, Ündar A. An evaluation of the benefits of pulsatile versus non-pulsatile perfusion during cardiopulmonary bypass procedures in pediatric and adult cardiac patients. ASAIO J. 2006;52:357-61.

24. Deakin CD, Barron DJ. Haemodynamic effects of descending aortic occlusion during cardiopulmonary resuscitation. Resuscitation. 1996;33:49-52.

25. Onorati F, Santarpino G, Tangredi G, Palmieri G, Rubino AS, Foti D, et al. Intraaortic balloon pump induced pulsatile perfusion reduces endothelial activation and inflammatory response following cardiopulmonary bypass. Eur J Cardiothorac Surg. 2009;35:1012-9. 